\title{
Oblique incidence sound absorption of parallel arrangement of multiple micro-perforated panel absorbers in a periodic pattern
}

Wang Chunqi ${ }^{*}$, Huang Lixi, Zhang Yumin

Lab of Aerodynamics and Acoustics, Zhejiang Institute of Research and Innovation and Department of Mechanical Engineering, The University of Hong Kong, Pokfulam Road, Hong Kong

*Corresponding author: E-mail: cqwang@hku.hk; chunqi76@gmail.com Tel: 852-2219 4547 


\begin{abstract}
The sound absorption performance of a micro-perforated panel (MPP) absorber array at oblique incidence and in diffuse field is investigated both numerically and experimentally. The basic module of the MPP absorber array consists of four parallel-arranged MPP absorbers with different cavity depths, and the whole MPP absorber array is created by arranging the basic modules in a periodically repeating pattern. A three-dimensional finite element model is used to simulate the acoustic properties of the MPP absorber array. Results show that the influence of incidence angle lies in two aspects. First, the parallel absorption mechanism breaks down at lower frequencies at oblique incidence than at normal incidence due to the non-compactness of the resonating MPP absorber, which becomes non-compact if the time delay of incident wave across it is comparable to or larger than $\pi / 2$. Second, the equivalent acoustic impedance of the MPP varies with respect to incidence angle which in turn changes the sound absorption performance of the MPP absorber array. Influence of the azimuthal angle is insignificant. It is also found that even the normal incidence sound absorption of the MPP absorber array differs from that of the basic module tested in impedance tube. The measured sound absorption coefficients of a prototype specimen in reverberation room compare well with the numerical predictions. The extra sound absorption due to diffraction of sound at the free edges of test specimen is the most efficient around $500 \mathrm{~Hz}$.
\end{abstract}

Keywords: sound absorption; micro-perforated panel; oblique incidence; parallel arrangement 


\section{Introduction}

Micro-perforated panels (MPP) have been successfully used in many noise control applications such as noise abatement in buildings [1, 2], medical devices [3], launcher fairings [4] and duct mufflers [5, 6]. A typical MPP absorber consists of an MPP and a rigid backing cavity. Though the MPP absorber constructed in this way performs much better than other resonant absorbers, its sound absorption capability is usually not quite enough as a general purpose absorber. Both its bandwidth and sound absorption coefficients are insufficient to compete with the fibrous materials. Recently, many efforts have been made to further enhance the sound absorption performance of MPP absorbers. Among them, one practical approach is to add additional MPPs into the backing cavity to form double-layer [7] or multiple-layer MPP absorbers [8, 9]. Investigations have also been reported to utilize the structural resonances of the MPP itself to improve its absorption performance of the MPP absorber, especially at low frequencies $[10,11]$.

As far as broadband absorption is concerned, one alternative and possibly more straightforward approach is to arrange multiple MPP absorbers of different frequency characteristics in parallel so as to combine different frequency bands together, hence an MPP absorber array. Previous studies on parallel arrangement of two different MPP absorbers [12-14] have shown great potential of the parallel arrangement to enhance its sound absorption performance. In a recent study by the present authors [15], the parallel absorption mechanism is identified to be contributed by three factors: (i) the strong local resonance absorption, (ii) the supplementary absorption by the non-resonating absorbers and (iii) the change of environmental impedance conditions; and the local resonance absorption mechanism accounts for the increased equivalent acoustic resistance of the MPP in the absorber array. The aforementioned research efforts are focused on the sound absorption of MPP absorber array at normal incidence of plane sound waves. In practical applications, however, the MPP is mainly subject to oblique sound waves, and its acoustic performance at oblique incidence 
may be quite different from that at normal incidence. The purpose of this work is to study how the parallel-arranged MPP absorber array performs at oblique incidence and in diffuse field.

In this paper, the oblique incidence sound absorption performance of the parallel-arranged MPP absorber array is investigated both numerically and experimentally. One major concern here is how the incidence angle of sound waves affects the parallel absorption mechanism. A finite element model is used to simulate the acoustic performance of an infinitely large MPP absorber array. One basic module of the MPP absorber array consists of a four-cavity configuration as shown in Fig. 1, and the whole MPP absorber array is created by arranging the basic four-cavity modules in a periodically repeating pattern. Results show that its sound absorption coefficients may change noticeably as the incidence angle varies. The diffuse field sound absorption coefficients of a prototype specimen are measured in a reverberation room and compared with the numerical predictions. The extra sound absorption in the experimental study is discussed by considering the diffraction effect due to the finite geometry of the test specimen.

\section{Theoretical modeling}

A three-dimensional (3D) configuration of the MPP absorber array is considered. Figure 1(a) shows one basic module of the MPP absorber array. The backing cavity is partitioned into four sub-cavities with different depth $\mathrm{D}_{1}, \mathrm{D}_{2}, \mathrm{D}_{3}$ and $\mathrm{D}_{4}$. The cavity walls and partitions are regarded as acoustically rigid. The MPP covering the air cavity can be either rigid or flexible, depending on the material and thickness of the panel. For some light-weight MPP, the effect of structural vibration can be significant at resonance frequencies $[10,16]$, but the effect of panel vibration is excluded in the present study for simplification. Assume that a plane sound wave $p_{\text {in }}$ is incident on the MPP with incidence angle $\theta$ and azimuthal angle $\beta$. Part of the incident sound energy is reflected or scattered, while the rest is absorbed by the MPP absorber. 


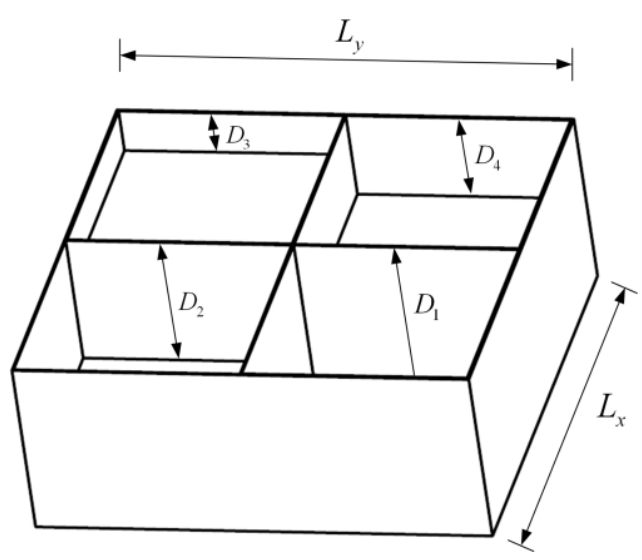

(a)

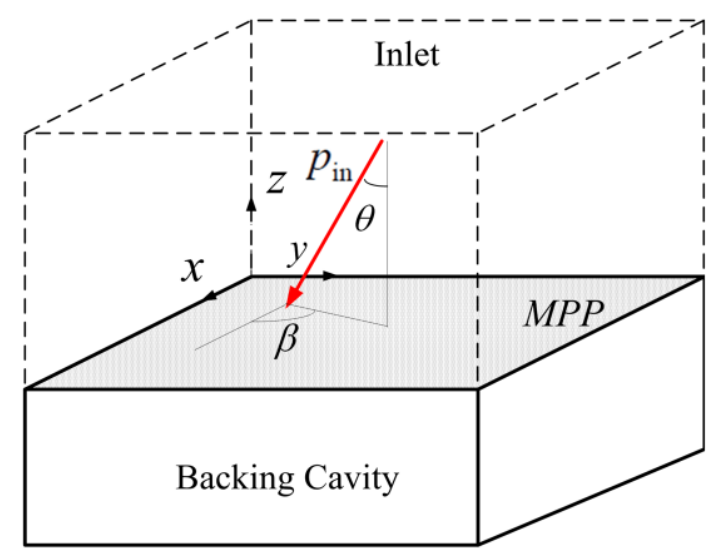

(b)

Figure 1. Configuration of the MPP absorber array. (a) Schematic of the partitioning of the backing cavity in one basic module; (b) Theoretical model of the MPP absorber array. The dashed lines represent the additional virtual duct for modeling the exterior sound field of the MPP absorber array.

A finite element procedure is used to simulate the acoustic performance of the MPP absorber array at oblique incidence in the frequency domain. The computational domain consists of the backing cavity (including the four sub-cavities), the MPP and a virtual duct with finite length as shown in Fig. 1(b). The finite element implementation of the 3D configuration is similar to that of a two-dimensional (2D) configuration described in [15], except that particular measures must be taken to deal with the boundary conditions of the virtual duct so that the scattered field can be simulated correctly. The sound field in the backing cavity and the virtual duct satisfies the Helmholtz equation

$$
\left(\nabla^{2}+k_{0}^{2}\right) \phi=0
$$

where $k_{0}=\omega / c_{0}$ is the wavenumber in free space, and $\phi$ is the velocity potential that is related to sound pressure $p$ and acoustic particle velocity $\mathbf{u}$ as

$$
p=-\mathrm{i} \rho_{0} \omega \phi, \quad \mathbf{u}=\nabla \phi .
$$

Here, $\rho_{0}$ and $c_{0}$ are the density and speed of sound in air; $\omega$ is the angular frequency; $i=\sqrt{-1}$ is the unit imaginary. By neglecting the structural vibration of the panel itself, the effect of MPP is implemented as a Neumann boundary condition 


$$
\frac{\partial \phi}{\partial z}=\frac{p_{\mathrm{cav}}-p_{\mathrm{duct}}}{\rho_{0} c_{0} Z}
$$

where $p_{\text {cav }}$ and $p_{\text {duct }}$ denote the sound pressure on the backing cavity side and on the virtual duct side respectively; $Z$ is the acoustic impedance of the MPP relative to air. Following Maa's formula [17], $Z$ can be found as

$$
Z=\frac{32 \eta t}{\sigma \rho_{0} c_{0} d^{2}}\left[\left(1+\frac{K^{2}}{32}\right)^{1 / 2}+\frac{\sqrt{2}}{32} K \frac{d}{t}\right]+\mathrm{i} \frac{\omega t}{\sigma c_{0}}\left[1+\left(9+\frac{K^{2}}{2}\right)^{-1 / 2}+0.85 \frac{d}{t}\right]
$$

where $K=d \sqrt{\omega \rho_{0} / 4 \eta}, d$ is the orifice diameter, $t$ is the panel thickness, $\eta$ is the coefficient of viscosity, and $\sigma$ is the perforation ratio in percentage.

A Dirichlet-to-Neumann (DtN) boundary condition [18] is applied at the inlet of the virtual duct so that the scattered sound wave can pass through this fictitious boundary without reflection. The implementation of the DtN boundary condition in this $3 \mathrm{D}$ model is described as below. The total sound wave at the inlet includes the plane incident sound wave and the scattered sound wave, that is,

$$
\phi=\phi_{\text {in }}+\phi_{\mathrm{sc}} .
$$

With the coordinate frame defined in Fig. 1(b), the incident sound wave is expressed as,

$$
\phi_{\text {in }}=\mathrm{e}^{\mathrm{i}\left(k_{x} x+k_{y} y+k_{z} z\right)}
$$

with the wavenumbers in the $x, y$ and $z$ directions being $k_{x}=k_{0} \sin \theta \cos \beta$, $k_{y}=k_{0} \sin \theta \sin \beta$ and $k_{z}=k_{0} \cos \theta$ respectively. The scattered sound wave is expressed in terms of modal expansion,

$$
\phi_{\text {sc }}=\phi-\phi_{\text {in }}=\sum_{m, n} A_{m n} \mathrm{e}^{\mathrm{i}\left(\kappa_{x, m} x+\kappa_{y, n} y-\kappa_{m n} z\right)}
$$

where $A_{m n}$ is unknown amplitude of the $(m, n)$ th mode of the scattered wave. Owing to the periodic conditions in the $x$ and $y$ directions, the wavenumbers of the $(m, n)$ th mode of the scattered wave are found as

$$
\kappa_{x, m}=k_{0} \sin \theta \cos \beta+2 m \pi / L_{x},
$$




$$
\begin{aligned}
& \kappa_{y, n}=k_{0} \sin \theta \sin \beta+2 n \pi / L_{y}, \\
& \kappa_{m n}=-\mathrm{i} k_{0} \sqrt{\left(\sin \theta \cos \beta+m \lambda / L_{x}\right)^{2}+\left(\sin \theta \sin \beta+n \lambda / L_{y}\right)^{2}-1},
\end{aligned}
$$

with $L_{x}, L_{y}$ being the side lengths of the rectangular backing cavity and $\lambda$ being the wavelength. At the inlet of the virtual duct $(z=0)$, the non-reflection requirement for the scattered wave leads to

$$
\frac{\partial \phi_{\mathrm{sc}}}{\partial z}=\sum_{m, n} A_{m n}\left(-\mathrm{i} \kappa_{m n}\right) \mathrm{e}^{\mathrm{i}\left(\kappa_{x, m} x+\kappa_{y, n} y\right)}
$$

From Eqs. (5), (6) and (9), the DtN boundary condition at the inlet is determined as

$$
\frac{\partial \phi}{\partial z}=\sum_{m, n} A_{m n}\left(-\mathrm{i} \kappa_{m n}\right) \mathrm{e}^{\mathrm{i}\left(\kappa_{x, m} x+\kappa_{y, n} y\right)}+\mathrm{i} k_{z} \mathrm{e}^{\mathrm{i}\left(k_{x} x+k_{y} y\right)}
$$

The unknown amplitude $A_{m n}$ is found as

$$
A_{m n}=\frac{1}{L_{x} L_{y}} \iint_{\mathbf{S}_{\text {Inlet }}}\left[\phi \mathrm{e}^{-\mathrm{i}\left(\kappa_{x, m} x+\kappa_{y, n} y\right)}-\mathrm{e}^{-\mathrm{i} 2 \pi \cdot\left(m x / L_{x}+n y / L_{y}\right)}\right] \mathrm{d} x \mathrm{~d} y .
$$

As the basic modules of the MPP absorber array are arranged in a periodically repeating pattern to create large sound absorption panels, the acoustic particle velocity and sound pressure at the front and back sides of the virtual duct satisfy the following periodic conditions:

$$
\begin{gathered}
\frac{\partial \phi_{B}}{\partial x}=\frac{\partial \phi_{F}}{\partial x} \mathrm{e}^{-\mathrm{i} k_{x} L_{x}} \\
\phi_{\mathrm{B}}=\phi_{\mathrm{F}} \mathrm{e}^{-\mathrm{i} k_{x} L_{x}},
\end{gathered}
$$

with the subscripts ' $\mathrm{B}$ ' and ' $\mathrm{F}$ ' referring to the back and front sides respectively. A similar periodic boundary condition applies to the left ('L') and right ('R') sides of the virtual duct,

$$
\begin{aligned}
& \frac{\partial \phi_{L}}{\partial y}=\frac{\partial \phi_{R}}{\partial y} \mathrm{e}^{-\mathrm{i} k_{y} L_{y}}, \\
& \phi_{L}=\phi_{\mathrm{R}} \mathrm{e}^{-\mathrm{i} k_{y} L_{y}} .
\end{aligned}
$$

The duct walls and partitions are assumed to be acoustically rigid, which means that the normal particle velocity vanishes in the normal direction $\mathbf{n}$ : 


$$
\mathbf{n} \cdot \nabla \phi=0
$$

The governing equation (1) and relevant boundary conditions (3), (10) and (12)-(14) are solved in a coupled manner using COMSOL Multiphysics ${ }^{\circledR}$ which is a commercial finite element software package for solving partial differential equations. The acoustic power absorbed by the MPP is then evaluated as,

$$
\begin{aligned}
P_{\text {abs }} & =-\iint_{\mathbf{S}_{\text {Inlet }}} \operatorname{Re}\left(p u_{z}^{*}\right) \mathrm{d} x \mathrm{~d} y \\
& =\rho_{0} \omega \iint_{\mathbf{S}_{\text {Illet }}} \operatorname{Re}\left[(\mathrm{i} \phi)(\partial \phi / \partial z)^{*}\right] \mathrm{d} x \mathrm{~d} y,
\end{aligned}
$$

where the asterisk denotes complex conjugate. For the incident sound wave $\phi_{\text {in }}$ defined in Eq. (6), the incident acoustic power on the MPP surface is,

$$
P_{\text {in }}=\rho_{0} \omega k_{0}(\cos \theta) L_{x} L_{y} .
$$

The oblique incidence sound absorption coefficient of the MPP absorber array is calculated as the ratio of $P_{\text {abs }}$ and $P_{\text {in }}$, that is,

$$
\alpha_{\theta, \beta}=\frac{\iint_{\mathbf{S}_{\text {Inlet }}} \operatorname{Re}\left[(\mathrm{i} \phi)(\partial \phi / \partial z)^{*}\right] \mathrm{d} x \mathrm{~d} y}{k_{0}(\cos \theta) L_{x} L_{y}}
$$

The sound absorption coefficient in diffuse field is then found as

$$
\alpha_{s}=\frac{1}{2 \pi} \int_{0}^{2 \pi}\left(\int_{0}^{\pi / 2} \alpha_{\theta, \beta} \sin 2 \theta \mathrm{d} \theta\right) \mathrm{d} \beta .
$$

Note that only a finite number of acoustic modes can be considered when implementing the DtN boundary condition defined by Eq. (10), that is, $-M \leq m \leq M$ and $-N \leq n \leq N$. Referring to Eqs. (7) and (8c), the (m,n)th acoustic mode may propagate within the virtual duct if the following condition is satisfied

$$
\left(\sin \theta \cos \beta+m \lambda / L_{x}\right)^{2}+\left(\sin \theta \sin \beta+n \lambda / L_{y}\right)^{2} \leq 1 .
$$

The above condition is used to calculate the critical frequency $f_{c}$ above which the $(m$, $n$ )th acoustic mode is cut-on. The number of acoustic modes necessary for the DtN boundary condition is thus determined by the geometrical size of the MPP absorber array and the frequency range of interest. Taking a square cross-section with 
$L_{x}=L_{y}=100 \mathrm{~mm}$ for example, the DtN boundary condition is exact up to $f_{c}=4200 \mathrm{~Hz}$ for random incidence when $M=N=3$.

\section{Numerical results}

The acoustic properties of an infinitely large MPP absorber array are investigated using the finite element model described in Section 2. The MPP absorber array is constructed by arranging the basic modules in Fig.1 in a periodically repeating pattern. The default values for the sub-cavity depths and MPP properties are listed in Table 1.

Table 1. The default settings for the sub-cavity depths and MPP properties.

\begin{tabular}{|c|c|c|c|c|c|c|}
\hline \multicolumn{4}{|c|}{ Depths of the sub-cavities } & \multicolumn{3}{|c|}{ MPP properties } \\
\hline$D_{1}$ & $D_{2}$ & $D_{3}$ & $D_{4}$ & $d$ & $t$ & $\sigma$ \\
\hline $100 \mathrm{~mm}$ & $50 \mathrm{~mm}$ & $12 \mathrm{~mm}$ & $25 \mathrm{~mm}$ & $0.6 \mathrm{~mm}$ & $0.6 \mathrm{~mm}$ & $1.6 \%$ \\
\hline
\end{tabular}

\subsection{Sound absorption at normal incidence $(\theta=0)$}

The normal incidence absorption performance of a sound absorption structure is usually simulated in a way as it would be tested experimentally in an impedance tube. For instance, if the four sides (Front, Back, Left and Right) of the virtual duct in Fig. 1(b) are assumed to be acoustically rigid instead of being periodic, the simulated normal incidence sound absorption coefficients will emulate those measured in an impedance tube. For a large MPP absorber array which consists of numerous basic modules arranged in a periodic repeating pattern, its normal incidence sound absorption performance can be noticeably different from those measured in an impedance tube because of mutual influence among the member MPP absorbers.

Figure 2 compares the predicted sound absorption coefficients of the above-mentioned two configurations. The legend "Periodic" denotes the configuration with the four sides of the virtual duct being periodic, corresponding to an infinitely large absorption panel. The legend "Rigid" refers to the configuration emulating the impedance tube. As analyzed in [15], the four sub-cavities and covering MPP form four resonators. In the periodic configuration, the sound absorption of each resonator 
is affected not only by the remaining three resonators within the same module but also by all other modules in the MPP absorber array, which in turn changes the acoustic performance of the MPP absorber array in comparison with the rigid configuration of the same geometrical dimensions. Actually, since the MPP absorber array is constructed in a periodically repeating manner, the effective geometrical dimensions of each basic module can be regarded as half the original one because of geometry symmetry. Figure 3(a) shows two adjacent basic modules in a large absorption panel, and each module consists of four sub-cavities indicated by different colors. At normal incidence, the large absorption panel can be regarded as being constructed by equivalent basic modules half the original one, as shown in Fig. 3(b). The four equivalent modules in Fig. 3(b) would have the same sound absorption properties if tested using an impedance tube. As shown in Fig. 2, the sound absorption of a rigid configuration with backing cavity of $L_{x}=L_{y}=100 \mathrm{~mm}$ is almost the same as that of a periodic configuration with backing cavity of $L_{x}=L_{y}=200 \mathrm{~mm}$.

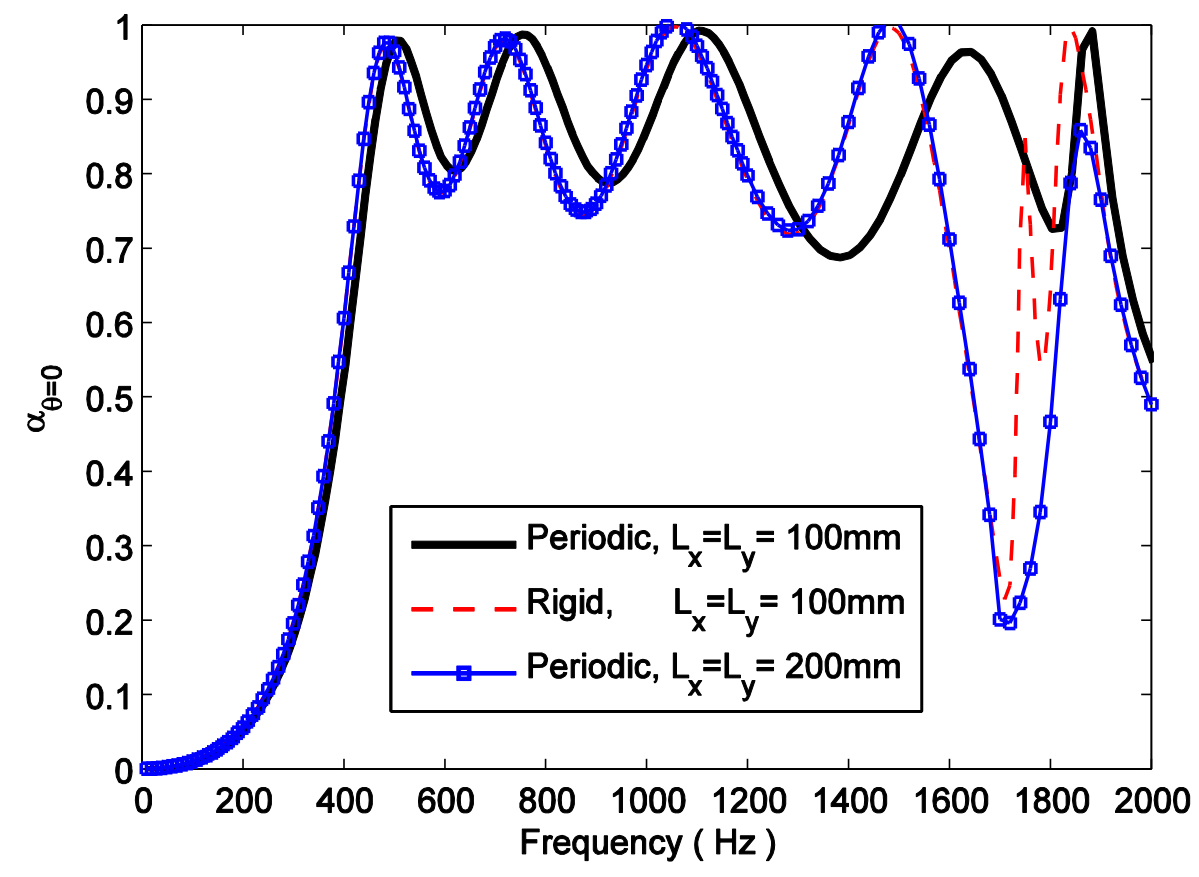

Figure 2: Comparison of the predicted normal incidence $(\theta=0)$ absorption coefficients between configurations with "periodic" and "rigid" boundary conditions on the four sides of the virtual duct. 


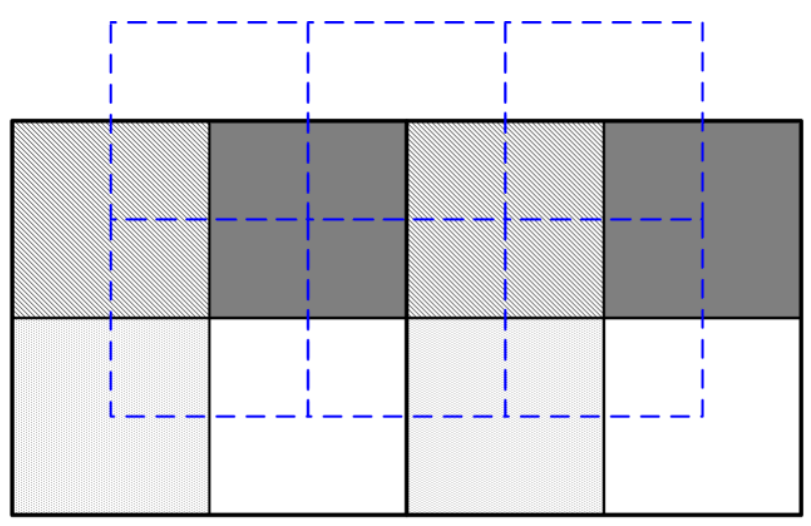

(a)

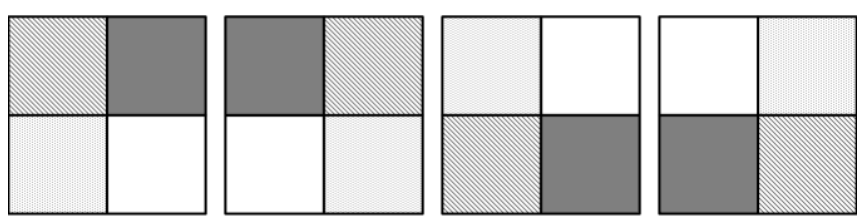

(b)

Figure 3: Illustration of the equivalent dimensions of the basic module arranged in a periodically repeating pattern. (a) Two adjacent basic modules in a large panel.

Each module consists of four sub-cavities with different depths. The dashed lines represent the equivalent basic modules with reduced size. (b) Four types of equivalent basic modules owing to the geometry symmetry.

The effect of geometrical size of the MPP absorber array on the normal incidence sound absorption is investigated by varying the values of $L_{x}$ and $L_{y}$, as shown in Fig. 4 . The results indicate that the parallel absorption mechanism works in an infinitely large MPP absorber array if the cavity width is less than the acoustic wavelength of interest. For example, for the backing cavity of $L_{x}=L_{y}=250 \mathrm{~mm}$, large absorption coefficients can be achieved up to $f=1350 \mathrm{~Hz}$, above which the absorption performance drops dramatically as the parallel absorption mechanism cannot sustain and the four resonators in the MPP absorber array respond to the incident sound wave in a separated way. For a larger backing cavity with $L_{x}=L_{y}=350 \mathrm{~mm}$, the parallel absorption mechanism breaks down at around $f=1000 \mathrm{~Hz}$. At oblique incidence, the parallel absorption mechanism fails faster than at normal incidence, and the reason is discussed in Section 3.3. 


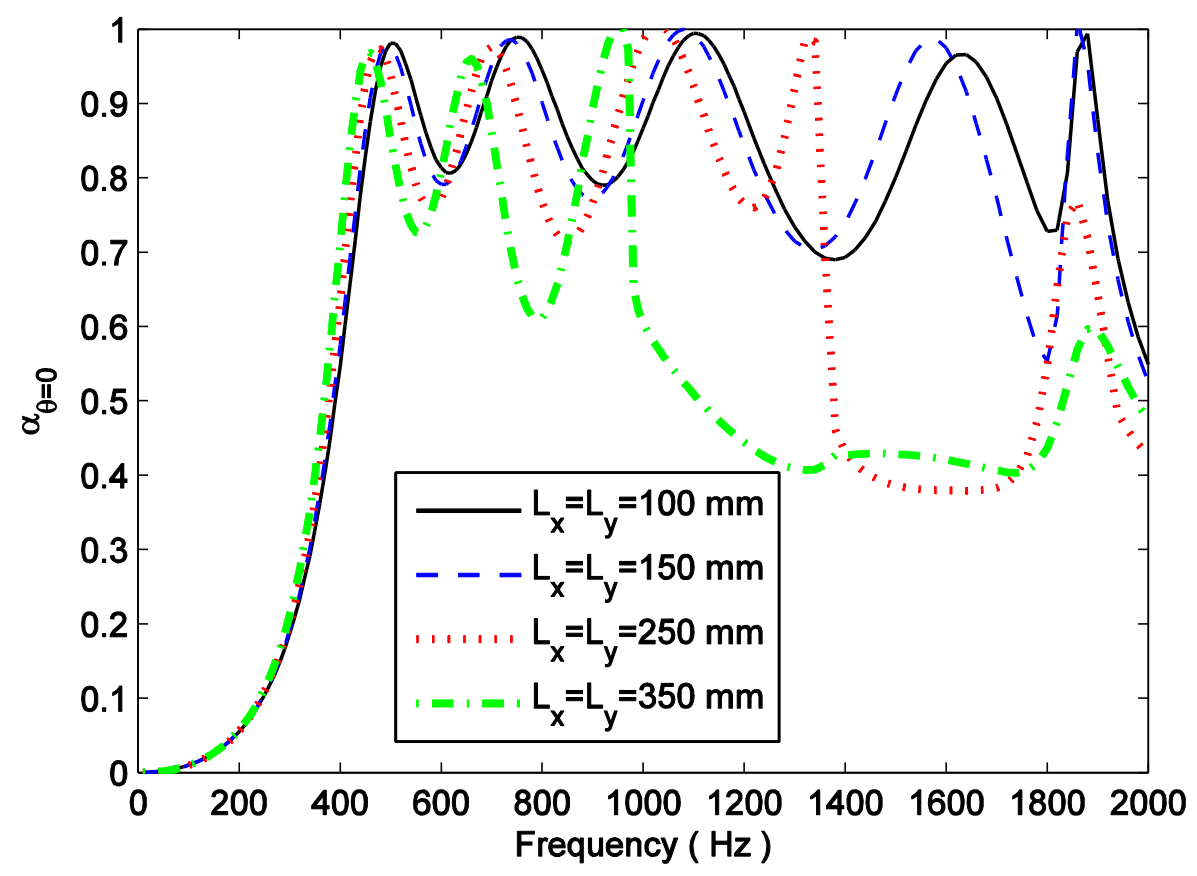

Figure 4: Variation of the normal incidence sound absorption coefficients of the MPP absorber array with respect to the geometrical dimension of backing cavity.

\subsection{Sound absorption at oblique incidence}

The influence of incidence angle $\theta$ on the sound absorption performance of the MPP absorber array is investigated by keeping the azimuthal angle $\beta$ constant. Figure 5 presents the overall profile of the sound absorption coefficient at different incidence angles from $\theta=0^{\circ}$ to $\theta=89^{\circ}$ with $\beta=90^{\circ}$. The sound absorption at grazing incidence $\left(\theta=90^{\circ}\right)$ cannot be dealt with using the present numerical model. It can be seen that the spectral shape of sound absorption curves remains almost the same as the incidence angle increases from $\theta=0^{\circ}$ to around $\theta=75^{\circ}$, which suggests that the parallel absortion mechanism works in a similar way for both oblique and normal incidences. At nearly grazing incence, say $80^{\circ}<\theta<90^{\circ}$, the sound absorption coefficient drops significantly, and the spectral peaks shift. The reasons for this phenomenon is explained below. 


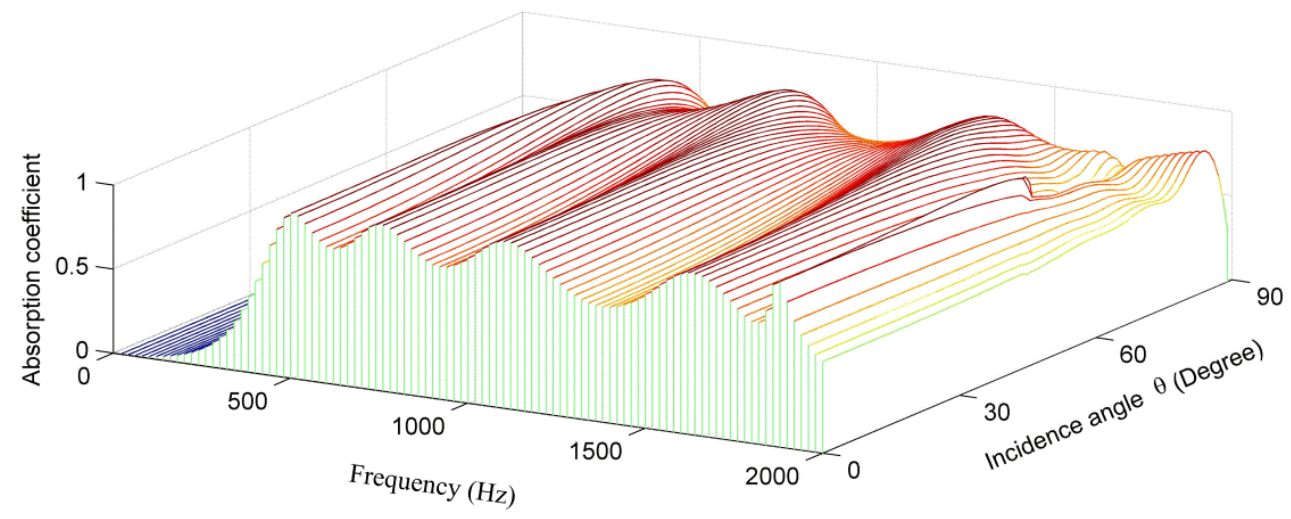

Figure 5. Variation of sound absorption coefficients at different incidence angles with $\beta=90^{\circ}$ and $L_{x}=L_{y}=100 \mathrm{~mm}$.

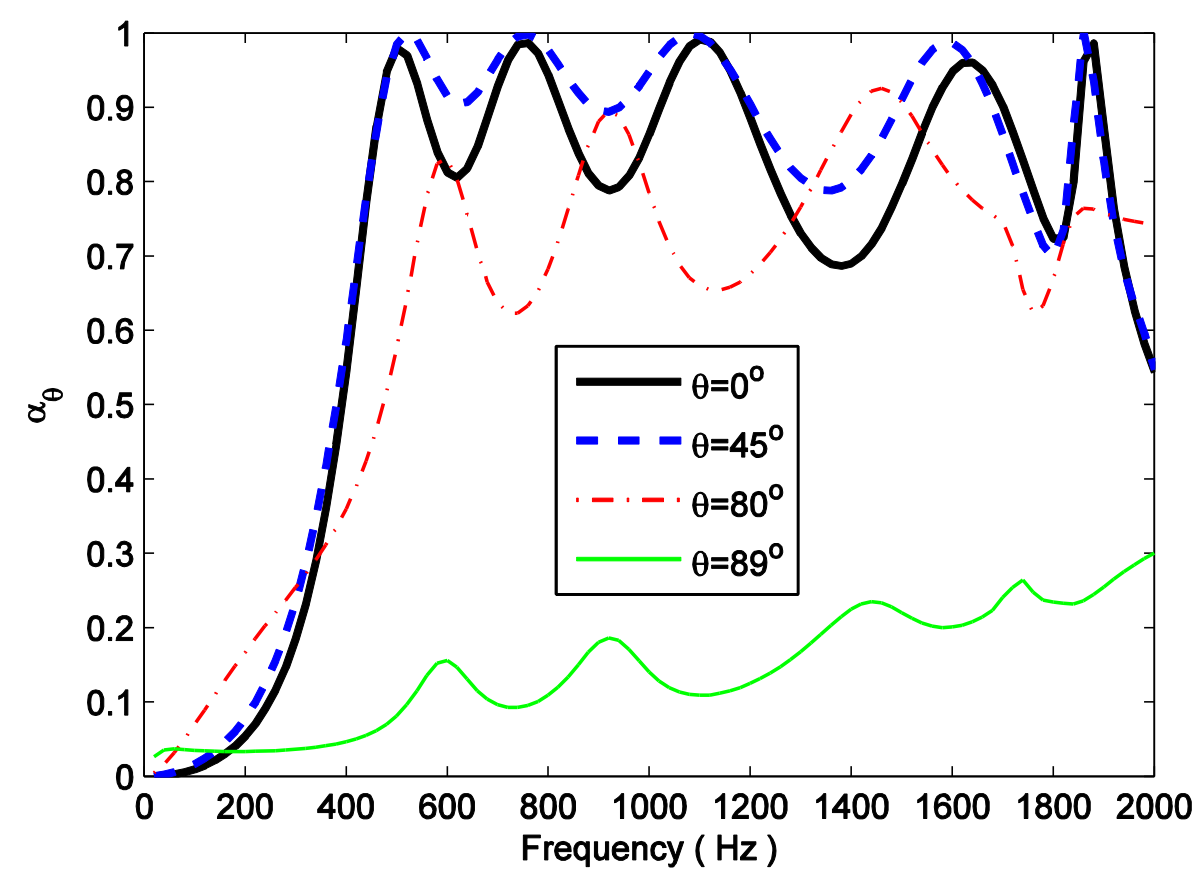

Figure 6. Comparison of sound absorption coefficients at different incidence angles with $\beta=90^{\circ}$ and $L_{x}=L_{y}=100 \mathrm{~mm}$.

The sound absorption coefficients at $\theta=0^{\circ}, 45^{\circ}, 80^{\circ}$ and $89^{\circ}$ are compared in Fig. 6 to better illustrate the influence of incidence angle. At normal incidece $\left(\theta=0^{\circ}\right)$, five spectral peaks are observed in the sound absorption curve within the frequency range considered. The first four peaks correspond to the resonances of the zeroth acoustic mode of the four sub-cavities with depth being $D=100 \mathrm{~mm}, 50 \mathrm{~mm}, 25 \mathrm{~mm}$ and 12 
$\mathrm{mm}$ respectively. The fifth spectral peak corresponds to the resonance of the first acoustic mode of the sub-cavity with $D=100 \mathrm{~mm}$. At $\theta=45^{\circ}$, the overall sound absorption coefficients increase significantly compared with those at normal incidence. There are still five resonance peaks in the absorption curve for $\theta=45^{\circ}$ but the resonance frequencies shift slightly. Further investigation shows that the acoustic response of the backing cavity at oblique incidence is still dominated by acoustic modes moving perpendicular to the MPP as at normal incidence. Acoustic modes that are moving parallel to the MPP may be excited at high frequencies which in turn causes extra resonance peaks in the absorption curve. Such a phenomenon can be observed for a larger backing cavity with $L_{x}=L_{y}=200 \mathrm{~mm}$, referring to Fig. 8. At nearly grazing incidence $\left(\theta=80^{\circ}\right.$ and $\left.89^{\circ}\right)$, the overall sound absorption coefficients drop dramatically, and the shift of resonance frequencies becomes more pronounced.

Numerical analyses show that the variation of sound absorption level and shift of resonance frequencies are mainly caused by the change of effective acoustic impedance of the MPP due to the incidence angle. As discussed in previous study on normal incidence sound absorption [15], around the resonance frequencies, the sound absorption is dominated by the resonance absorption of one MPP absorber, and the sound absorption by the rest three non-resonating absorbers is trivial. Therefore, for the four-cavity configuration in Fig. 1(a), it is reasonable to assume that the effective MPP area is approximately $1 / 4$ of the total area. In other words, the effective perforated area is $1 / 4$ of the total MPP area, and the equivalent perforation ratio $\sigma^{\prime}=\sigma / 4$ with $\sigma$ being the nominal perforation ratio of the MPP. As a result, the effective acoustic impedance of the MPP, $Z^{\prime}$, is approximately four times the value calculated using Eq. (4) in which $\sigma$ instead of $\sigma$ is used. When the sound wave impinges on the MPP with an incidence angle $\theta$, the corresponding effective acoustic impedance then becomes $Z^{\prime} \cos \theta$. Figure 7 presents the effective acoustic resistance of the MPP at different incidence angles. The acoustic impedance of air $\left(Z_{\text {air }}=1\right)$ is shown for reference. The effective acoustic impedance of the MPP diminishes as the incidence angle increases. Note that the effective acoustic resistance of the MPP at 
$\theta=45^{\circ}$ matches the impedance of air better than at normal incidence for the given MPP parameters, which explains the enhanced sound absorption performance at $\theta=45^{\circ}$ as observed in Fig. 6. At nearly grazing incidence, the effective acoustic resistance of the MPP becomes so small compared to air that good absorption performane cannot be achieved. The equivalent acoustic reactance of the MPP also decreases as the incidence angle increases, which generally shifts the resonance frequencies to higher values.

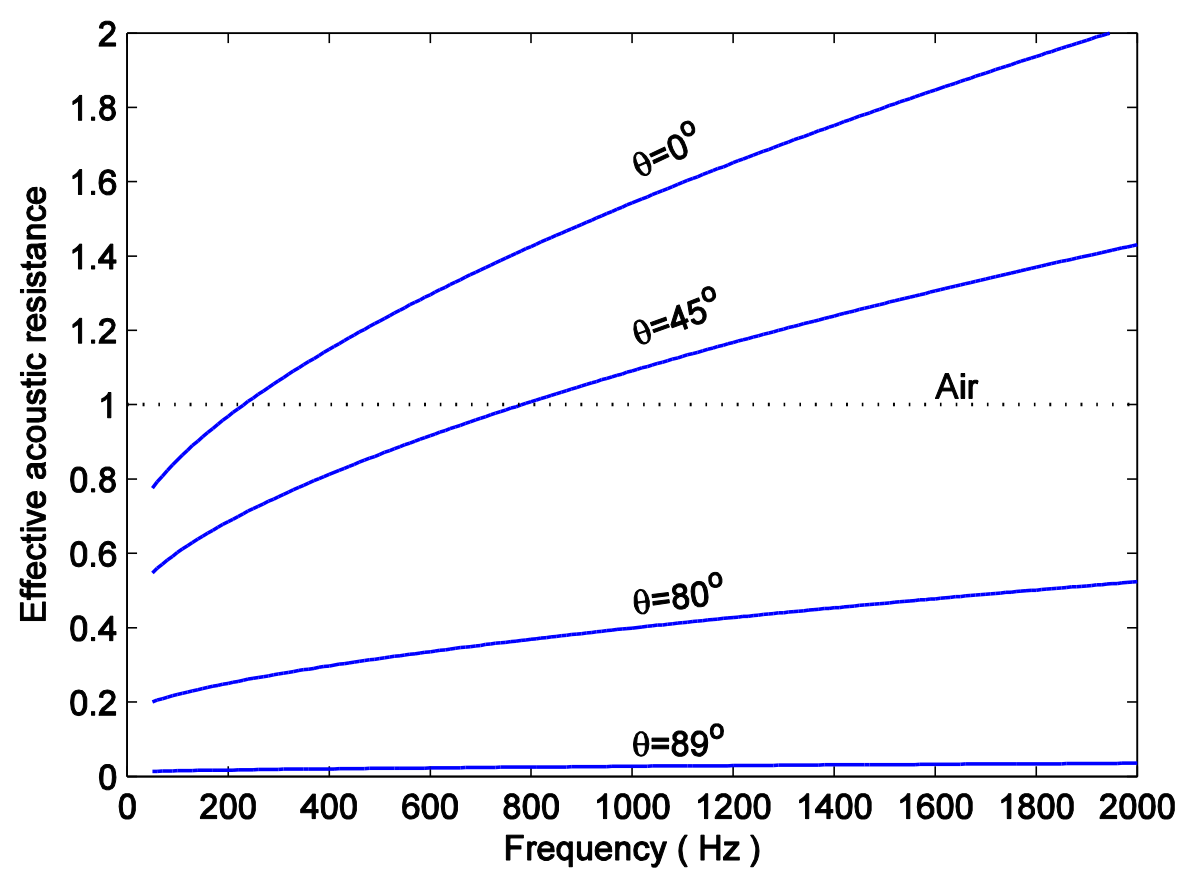

Figure 7: The effective acoustic resistance of the MPP at different incidence angles. The effective acoustic resistance is estimated as four times the acoustic resistance calculated by Eq. (4).

In this study, it is found that the parallel absorption mechanism of the MPP absorber array breaks down at lower frequency at oblique incidence than at normal incidence. Figure 8 shows the variation of the sound absorption coefficients of the MPP absorber array at $\theta=45^{\circ}$ and $\beta=90^{\circ}$. Take the backing cavity with $L_{x}=L_{y}=150$ mm for example. A sudden drop can be observed in the sound absorption curve at $f=1400 \mathrm{~Hz}$, which suggests that the parallel absorption mechanism breaks down above that frequency. At normal incidence, the parallel absorption mechanism works up to $f=2000 \mathrm{~Hz}$ for the same configuration. As far as the applications at oblique incidence 
or random incidence are concerned, therefore, the size of the backing cavity should be smaller than that at normal incidence to achieve the parallel sound absorption effect.

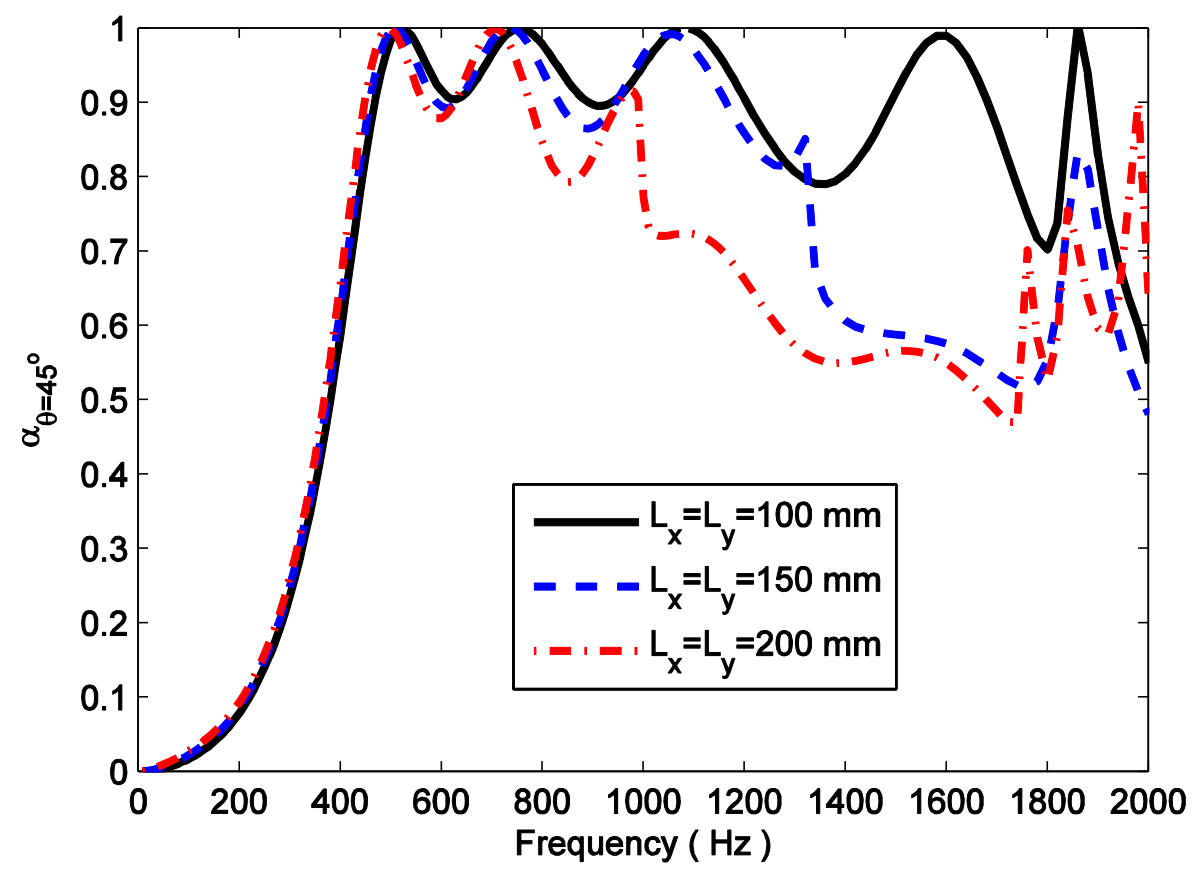

Figure 8: Variation of the oblique incidence sound absorption coefficients with respect to the geometrical dimensions of the backing cavity. $\theta=45^{\circ}$ and $\beta=90^{\circ}$.

The effect of azimuthal angle on the absorption of the MPP absorber array is examined by keeping the incidence angle $\theta=45^{\circ}$. Two configurations with different backing cavity sizes are considered and the results are presented in Fig. 9. One observation here is that the effect of azimuthal angle on the sound absorption is very insignificant as long as the parallel absorption mechanism can be maintained, referring to Fig. 9(a). In the high frequency range in Fig. 9(b), the parallel mechanism breaks down, and the sound absorption coefficient changes noticeably with respect to the azimuthal angle.

The sound absorption coefficients in diffuse field are calculated through numerical integration based on Eq. (18). The results for three types of backing cavities are shown in Fig. 10. The diffuse field incidence absorption curves are 
comparable to those at incidence angle $\theta=45^{\circ}$. The oblique incidence absorption coefficient for $L_{x}=L_{y}=150 \mathrm{~mm}$ at $\theta=45^{\circ}$ is also shown in Fig. 10 for reference. Note that the finite element model simulates an infinitely large MPP absorber array. For a finite MPP absorber array, the sound absorption coefficients can be higher than those presented in this figure due to the diffraction of sound at the edges of the absorbent surface [19].

(a)

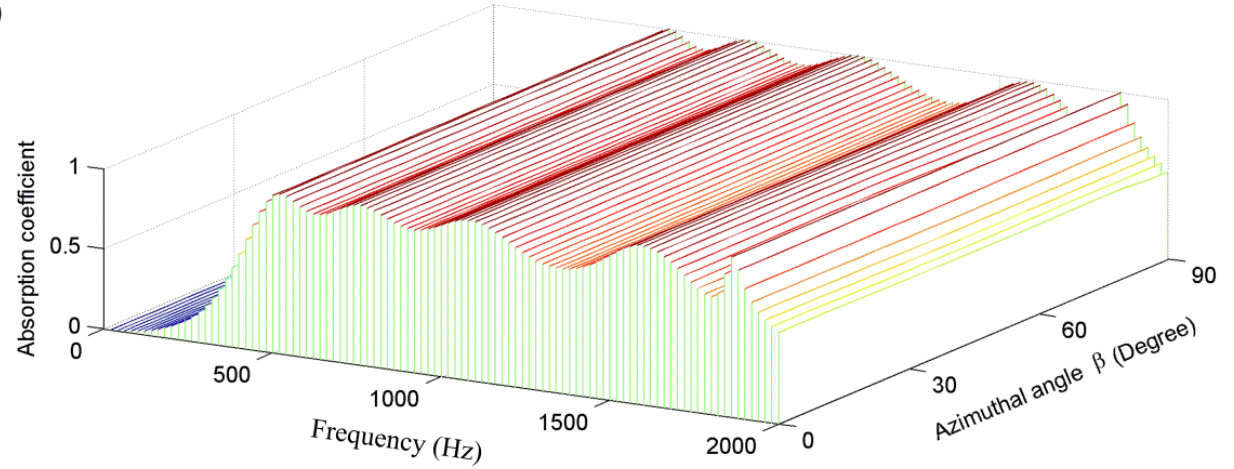

(b)

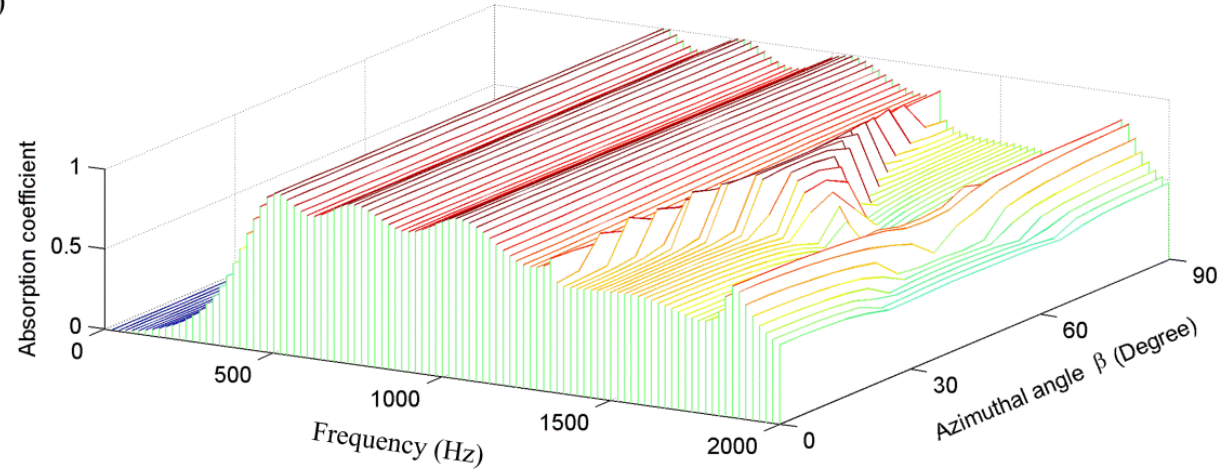

Figure 9: Variation of sound absorption coefficients at different azimuthal angles with $\theta=45^{\circ}$. (a) $L_{x}=L_{y}=100 \mathrm{~mm}$; (b) $L_{x}=L_{y}=200 \mathrm{~mm}$. 


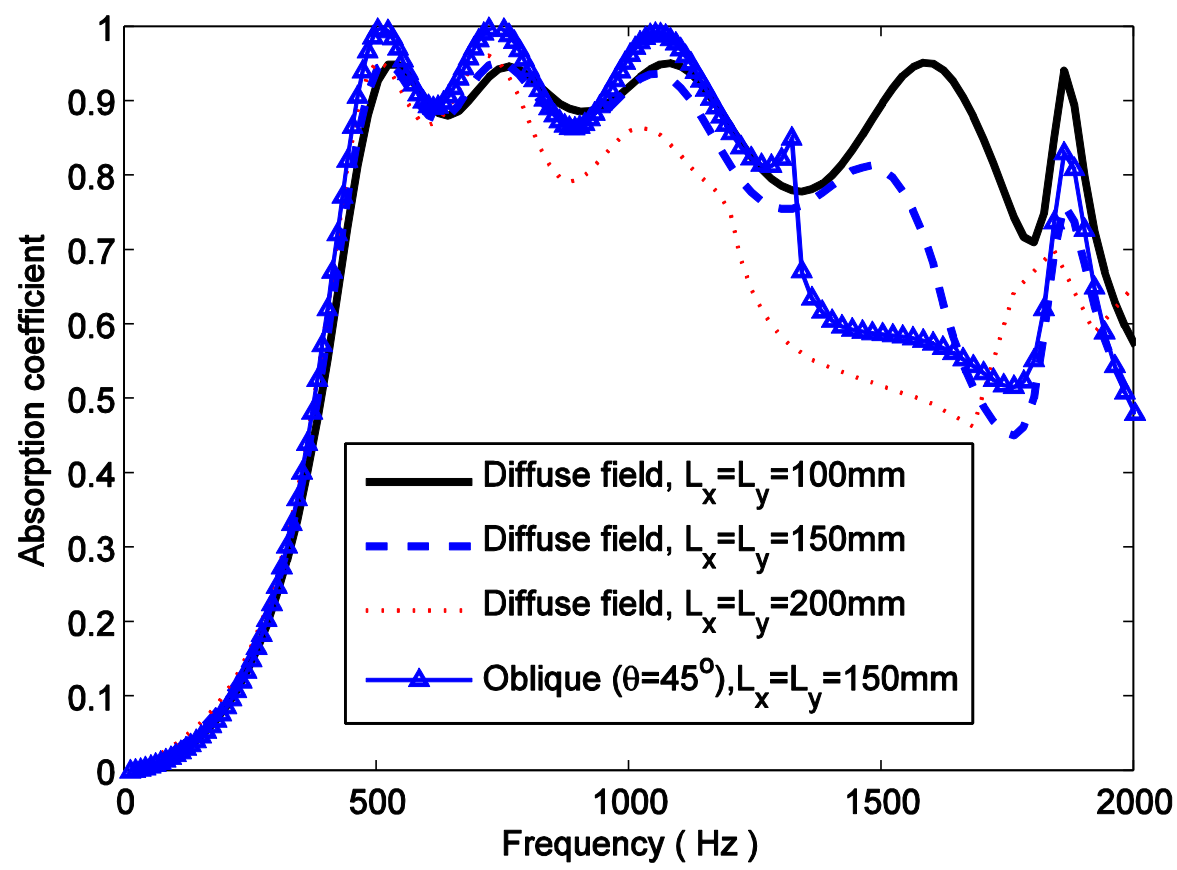

Figure 10: Predicted diffuse field sound absorption coefficients for different geometrical dimensions of the backing cavity. The oblique incidence absorption coefficient for $L_{x}=L_{y}=150 \mathrm{~mm}$ at $\theta=45^{\circ}$ is shown for reference.

\subsection{Analyses on the failure of parallel absorption mechanism at oblique incidence}

Comparisons between Figs. 4 and 8 indicate that the parallel absorption mechanism breaks down at lower frequencies at oblique incidence than at normal incidence. This phenomenon is analyzed below. Note that the four MPP absorbers in Fig. 1 interact with each other, which complicates the analyses unnecessarily. For simplicity, a two-cavity configuration is considered with the cavity depths being $D_{1}=100 \mathrm{~mm}, D_{2}=25 \mathrm{~mm}$, which is simplified to a 2D problem as in Fig. 11(a). The simulated sound absorption coefficients at $\theta=45^{\circ}$ and $60^{\circ}$ are shown in Fig. 11(b). When $L=100 \mathrm{~mm}$, the parallel absorption mechanism breaks down around $f=1050 \mathrm{~Hz}$ when $\theta=45^{\circ}$, and the breakdown occurs at lower frequency $f=960 \mathrm{~Hz}$ when $\theta=60^{\circ}$.

The acoustic field of the absorber array is examined to explore the reason for the breakdown of parallel absorption mechanism. Results show that, at oblique incidence, the parallel absorption mechanism is still dominated by the local resonance absorption, but the non-compactness of the resonating MPP absorber may change the absorption 
pattern and then cause a faster breakdown of the parallel absorption mechanism than at normal incidence. For illustration purposes, the sound intensity distributions at frequencies $f=800 \mathrm{~Hz}$ and $1060 \mathrm{~Hz}$ with $\theta=45^{\circ}$ are shown in Fig. 12. The resonance absorption by cavity II with depth $D_{2}=25 \mathrm{~mm}$ is dominant in the sound absorption at both frequencies, but the absorption patterns are different. At $f=800 \mathrm{~Hz}$, the parallel absorption mechanism works and the incident acoustic energy is attracted to cavity II and then dissipated. The energy dissipation almost occurs in the whole region of the MPP covering cavity II, and the resonating MPP absorber works like a locally-reacting impedance surface, just as in the case at normal incidence. At $f=1060$ $\mathrm{Hz}$, however, the energy dissipation occurs only on a small portion of the MPP, and significant energy transport is observed inside cavity II. As shown in Fig. 12, the acoustic energy enters cavity II from the left side and goes out from the right side. Though the sound absorption is still mainly generated by the resonance of cavity II, the resonating MPP absorber (cavity II and the covering MPP) is no longer locally reacting and the parallel absorption mechanism fails to work.

The compactness of the resonating MPP absorber is roughly measured by the time delay of the incident wave across it,

$$
\Omega=k_{0} L \sin \theta
$$

It is observed that the compactness requirement is no longer satisfied when $\Omega$ is comparable to or larger than $\pi / 2$ and, as a result, the parallel absorption mechanism breaks down. In other words, the width of the sub-cavity should be less than $\lambda_{\text {min }} /(4 \sin \theta)$ where $\lambda_{\text {min }}$ is the wavelength of the critical frequency below which the parallel absorption mechanism is expected to work. Meanwhile, Eq. (20) suggests that the breakdown at oblique incidence may be avoided or shifted to higher frequency by reducing the cavity width. For example, the breakdown frequency is increased from $960 \mathrm{~Hz}$ to $1160 \mathrm{~Hz}$ at $\theta=60^{\circ}$ when the cavity width is reduced from $L$ $=100 \mathrm{~mm}$ to $80 \mathrm{~mm}$. Recalling the observations on normal incidence, the width of the sub-cavity is suggested to be smaller than $\lambda_{\min } /(4 \sin \theta)$ or $\lambda_{\min }$, whichever is 
smaller, to achieve the parallel absorption mechanism. Note that no particular effort is made to seek optimal performance of the MPP absorber array at the present stage. In practice, its absorption performance can be maximized by choosing an optimal set of parameters, including both the geometrical size of sub-cavities and perforation properties of the MPP. Toward this purpose, a global optimization method such as the genetic algorithms [20] may be adopted to avoid the searching process trapped in a suboptimal local maximum or minimum of the target function.

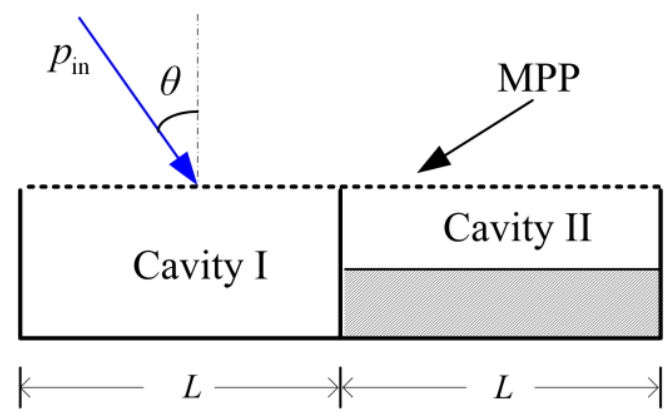

(a)

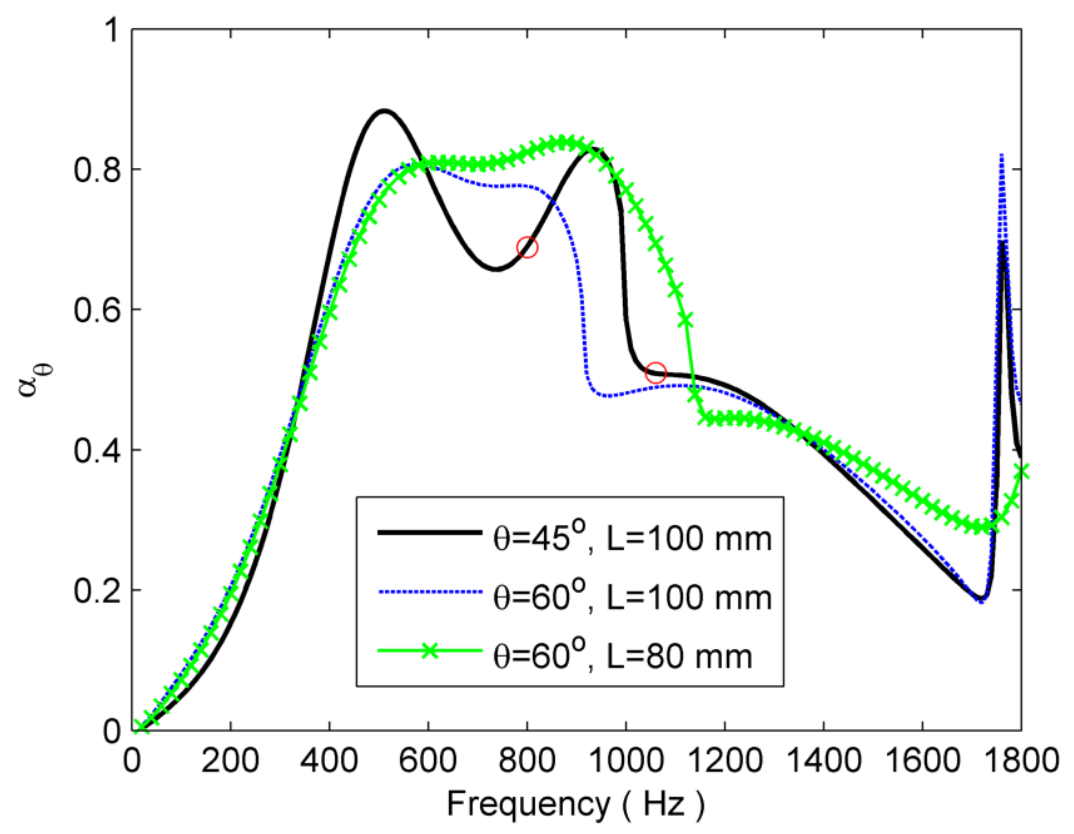

(b)

Figure 11: Oblique incidence sound absorption of parallel arrangement of two MPP absorbers with different cavity depths (100 mm and $25 \mathrm{~mm}$ ). (a) Schematic of the 2D configuration; (b) Sound absorption coefficients at different incidence angles. 

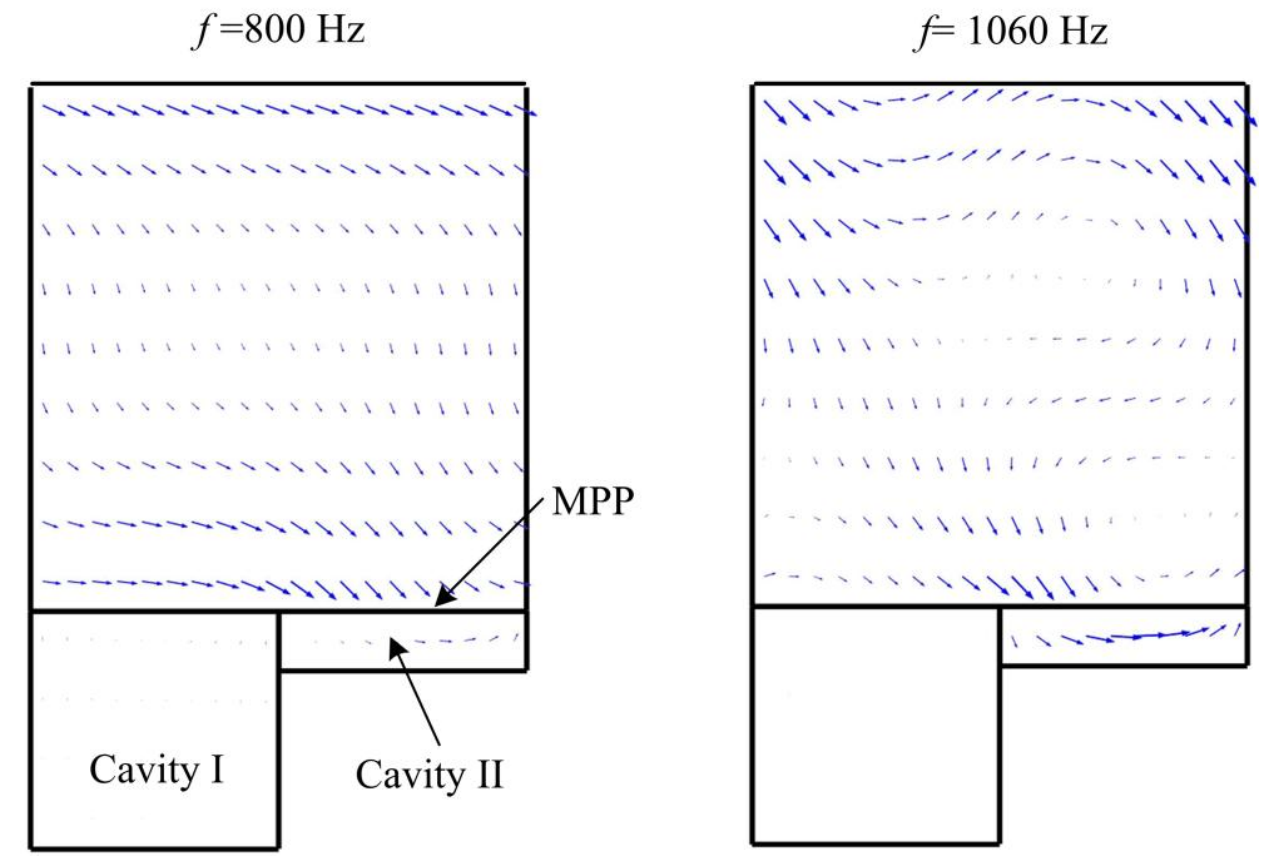

Figure 12: Distributions of the sound intensity vectors below and above the breakdown frequency $(f=1050 \mathrm{~Hz})$ of the parallel absorption mechanism. The arrow length indicates the relative magnitude of sound intensity.

\subsection{Comparison with conventional MPP absorbers}

The MPP absorber array in Fig. 1 becomes a conventional MPP absorber if the depths of the four sub-cavities are set to be the same. In this situation, the MPP absorber may be treated as a locally reacting surface, and the oblique incidence sound absorption coefficient can be estimated through a simple analytical formula as in Eq. (21a). If the backing cavity is treated as a uniform air gap without partition, path difference between the incident and reflected waves from the backing wall changes with the incidence angle, and the oblique incidence sound absorption coefficient is calculated using Eq. (21b) [7],

$$
\begin{aligned}
& \alpha_{\theta}=\frac{4 \operatorname{Re}\left(Z_{\mathrm{MPPA}}\right) \cos \theta}{\left[1+\operatorname{Re}\left(Z_{\mathrm{MPPA}}\right) \cos \theta\right]^{2}+\left[\operatorname{Im}\left(Z_{\mathrm{MPPA}}\right) \cos \theta\right]^{2}}, \\
& \alpha_{\theta}=\frac{4 \operatorname{Re}(Z) \cos \theta}{[1+\operatorname{Re}(Z) \cos \theta]^{2}+\left[\operatorname{Im}(Z) \cos \theta-\cot \left(\omega D \cos \theta / c_{0}\right)\right]^{2}},
\end{aligned}
$$

where $Z$ is the relative acoustic impedance of the MPP calculated by Eq. (4), $D$ is the 
depth of the backing cavity, $Z_{\mathrm{MPA}}=Z-\mathrm{i} \cot \left(\omega D / c_{0}\right)$ is the acoustic impedance on the external surface of the MPP. Equations (21a) and (21b) are for conventional MPP absorbers with partitioned and non-partitioned backing cavity, respectively. The oblique incidence absorption coefficients of an MPP absorber with $D=100 \mathrm{~mm}$ calculated by Eqs. (21a) and (21b) are plotted in Fig. 13. Results by the 3D finite element model in the current study are also given for comparison. In the finite element model, the size of the sub-cavity is assumed to be $L_{x}=L_{y}=100 \mathrm{~mm}$. Figure 13 shows that results by the present finite element model compare well those by Eq. (21a). For the non-partitioned case, the whole absorption curve is shifted to higher frequencies since the effective cavity depth is decreased.

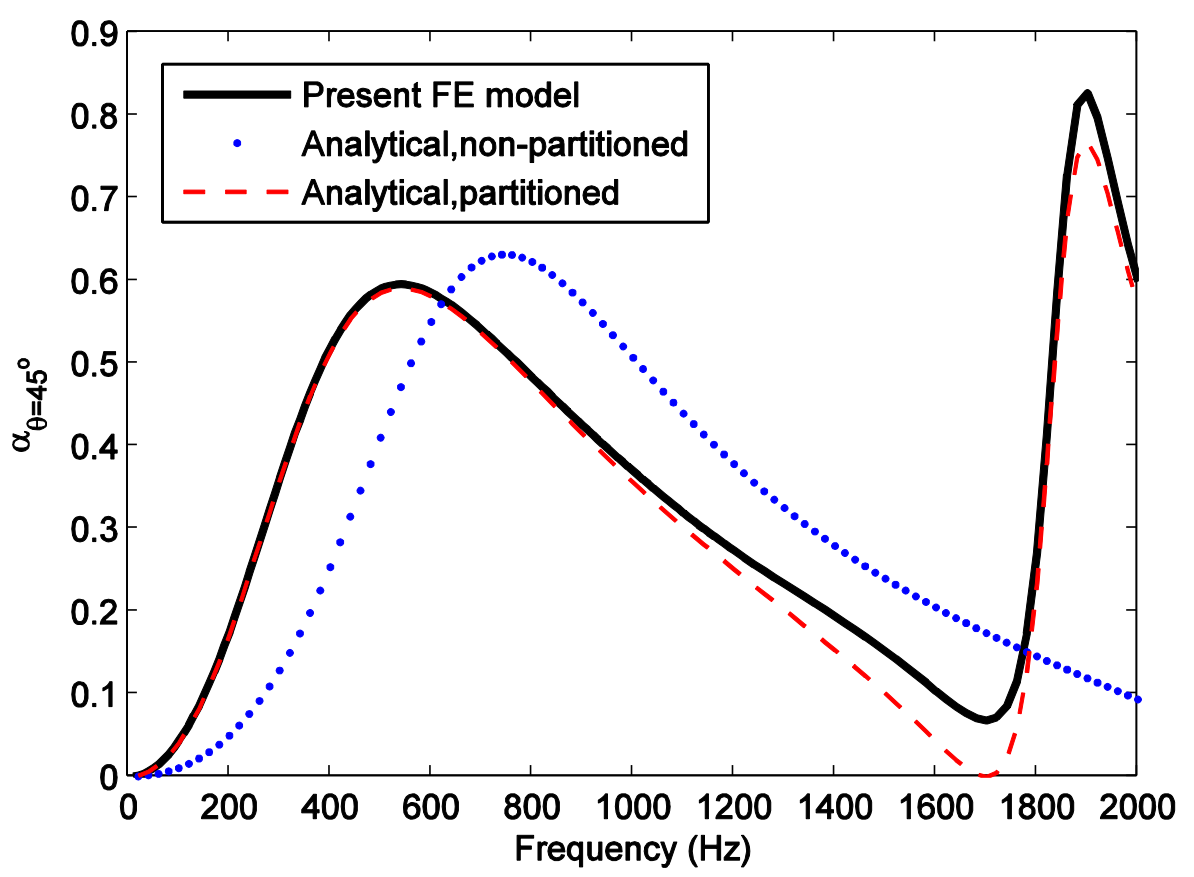

Figure 13: Sound absorption coefficients of a conventional uniform MPP absorber predicted by three models. Incidence angle $\theta=45^{\circ}$, cavity depth $D=100 \mathrm{~mm}$. Other parameters are given in Table 1.

It is of interest to check whether Eq. (21a) can be applied to the present four-cavity MPP absorber array to estimate its oblique incidence absorption coefficients. In doing so, the surface acoustic impedance $Z_{\mathrm{MPPA}}$ in Eq. (21a) is 
replaced by an equivalent surface acoustic impedance $Z_{\mathrm{MPPA}}^{\prime}$ which is determined as

$$
\frac{1}{Z_{\mathrm{MPAA}}^{\prime}}=\frac{1}{4} \sum_{j=1}^{4} \frac{1}{Z-\mathrm{i} \cot \left(\omega D_{j} / c_{0}\right)} .
$$

The default parameters specified in Table 1 is used. Results by the analytical formula (Eq. (21a)) and the present 3D finite element model are compared in Fig. 14. Significant differences can be observed between the two methods, especially when the geometrical size of the backing cavity is large. Note that the sound absorption performance of the MPP absorber array is a function of incidence angle, azimuthal angle, geometrical size of the sub-cavity, etc. However, Eq. (21a) only considers the effect of incidence angle and the influence by other factors are omitted. Actually, Eq. (21a) represents the extreme situation in which the size of sub-cavity is infinitesimal compared with the acoustic wavelength. Therefore, the sound absorption coefficients of the present MPP absorber array may be roughly estimated using Eq. (21a), but accurate numerical models like the one in this study are necessary for its actual acoustic performance.

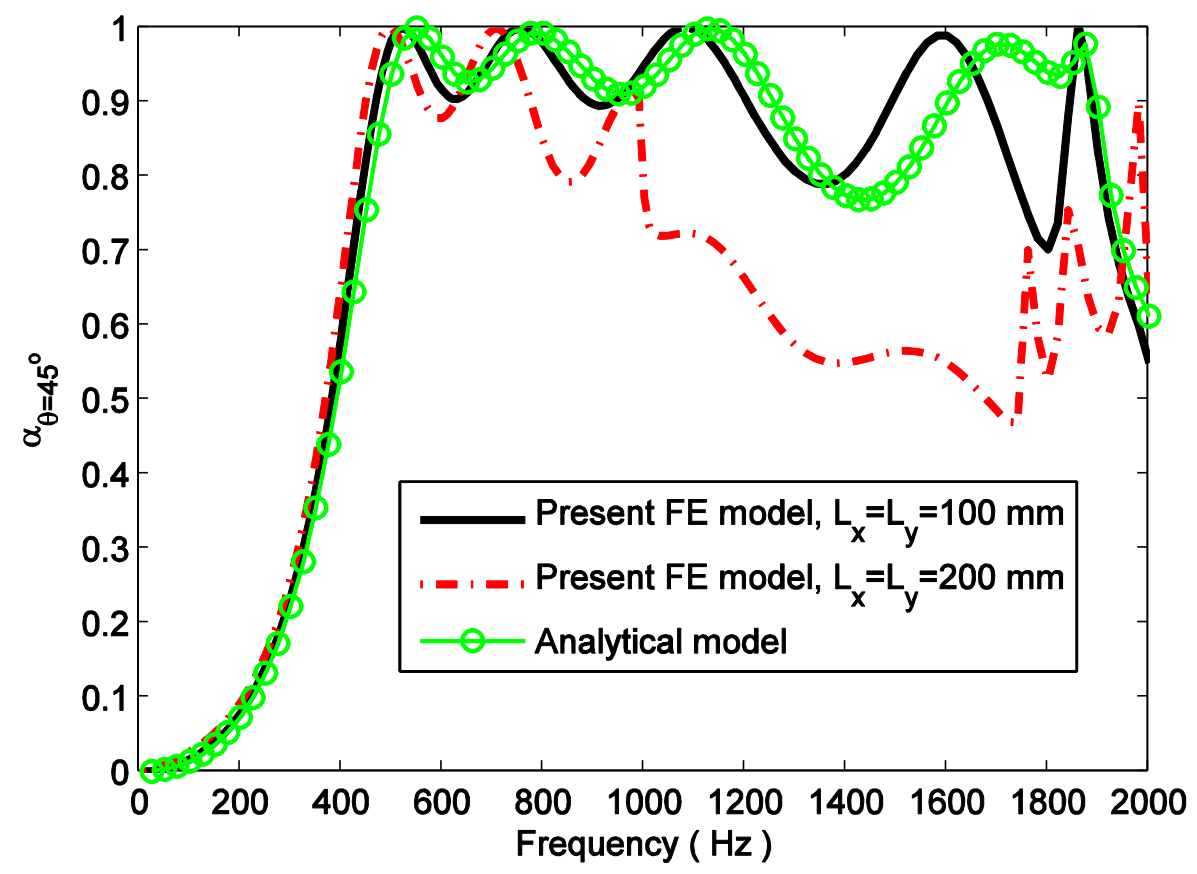

Figure 14: Predicted oblique incidence sound absorption coefficients of the four-cavity MPP absorber array specified in Table 1. $\theta=45^{\circ}$ and $\beta=90^{\circ}$. 


\section{Measurement in reverberation room}

The sound absorption coefficient of a parallel-arranged MPP absorber array is measured in a reverberation room and compared with the numerical predictions. The reverberation room has a volume of $275 \mathrm{~m}^{3}$ and a floor area of $45 \mathrm{~m}^{2}$, which provides a diffuse sound field for the experimental tests. The measurements are made following the interrupted noise method as specified in the ISO 354:2003 standard [21]. The test specimen has a four-cavity configuration as shown in Fig. 1 with $L_{x}=L_{y}=200 \mathrm{~mm}$. The total area of the test specimen is $11.52 \mathrm{~m}^{2}$. The cavity walls are made of wood plate of thickness of $10 \mathrm{~mm}$; and the backing cavity is partitioned into sub-cavities with depths $D_{1}=100 \mathrm{~mm}, D_{2}=50 \mathrm{~mm}, D_{3}=12 \mathrm{~mm}, D_{4}=25 \mathrm{~mm}$ with wood plate of thickness of $4 \mathrm{~mm}$. The three shallow sub-cavities are created by inserting PVC foams into sub-cavities of $\mathrm{D}_{1}=100 \mathrm{~mm}$. The parameters of the MPP covering the backing cavity are $d=0.5 \mathrm{~mm}, t=0.5 \mathrm{~mm}, \sigma=1.6 \%$. The MPP is made of steel, and the perforations are formed through mechanical punching. As shown in Fig. 15, the test specimen is placed directly against the floor of the reverberation chamber; and the perimeter edges of the test specimen are covered with acoustically reflective plates to prevent the edges from absorbing sound.

The diffuse field sound absorption coefficient of the test specimen is measured at the center frequencies of one-third octave bands from $100 \mathrm{~Hz}$ to $5000 \mathrm{~Hz}$. The measured results in the frequency range of $100 \mathrm{~Hz}$ to $2000 \mathrm{~Hz}$ are shown in Fig. 16 by the solid curve with open circles. Owing to the low modal density of the reverberation chamber, the results at low frequencies, say below $200 \mathrm{~Hz}$, are not reliable. The solid curve is for the simulated sound absorption coefficients of an infinitely large geometry. The measured results are in agreement with the numerical predictions in general, while the measured values are higher than the predictions by $0.1-0.3$. The maximum measured sound absorption coefficient is $\alpha_{s}=1.2$ at $500 \mathrm{~Hz}$. Several mechanisms may account for the increase of sound absorption of the test specimen compared with the numerical predictions, for examples, the excess sound absorption by the wood frames, 
the vibration of the MPP itself, and the so-called edge effect. The edge effect is a diffraction phenomenon along the free edges of the test specimen, which causes additional sound energy flow inwards to the specimen from all around. In other words, the effective absorption area is acoustically larger than the geometrical area of the specimen. Owing to the edge effect, the absorption coefficient measured in a reverberation room can be larger than 1 as the geometrical area is used in the calculation of the absorption coefficient. More information on the edge effect can be found in $[22,23]$.

Since edge effect is usually the major contributor to the increase of sound absorption measured in reverberation rooms, a rough estimation of its influence is made below. For the measurement in reverberation rooms, previous experimental studies have shown that a linear relationship may be assumed between the measured absorption coefficient and the relative edge length of the test specimen [19],

$$
\alpha_{s}^{\prime}=\alpha_{s}+\gamma E,
$$

where $\alpha_{s}$ is the measured absorption coefficient of the finite test specimen in reverberation room, $\alpha_{s}$ is the absorption coefficient of an infinite large absorptive panel, $\gamma$ is a constant, and $E$ is the relative edge length of the test specimen. Equation (23) shows that the absorption coefficient of a finite test specimen can be estimated based on the predicted absorption coefficient of an infinitely large configuration when the constant $\gamma$ is known. If the MPP absorber array is treated as a locally reacting surface of acoustic impedance $Z_{\mathrm{MPPA}}^{\prime}$ in Eq. (22), the absorption coefficient of the finite specimen can be predicted using boundary element method with reasonable accuracy [24], and the constant $\gamma$ can then be determined. The dashed line in Fig. 16 shows the estimated absorption coefficient with edge effect. It can be observed that the edge effect is the most efficient in the neighborhood of $500 \mathrm{~Hz}$, where the extra absorption coefficient due to edge effect is of the order of 0.25 . 


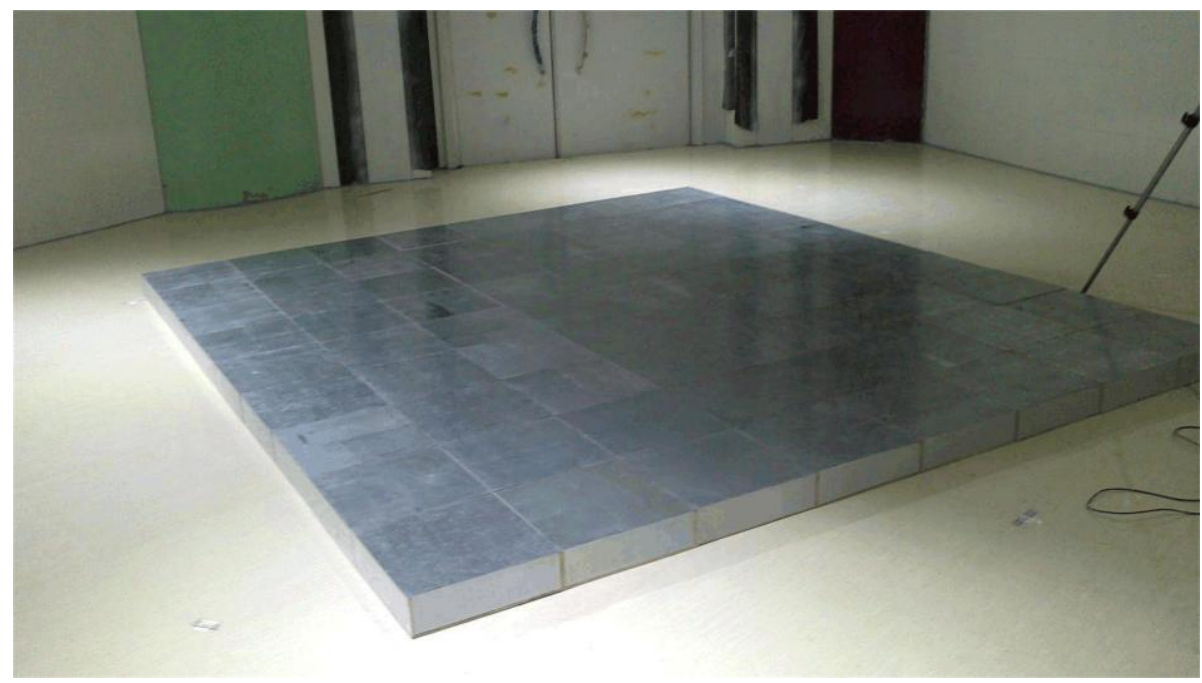

Figure 15: Photo of the large test specimen in the reverberation chamber. The large test specimen consists of 72 pieces of square samples.

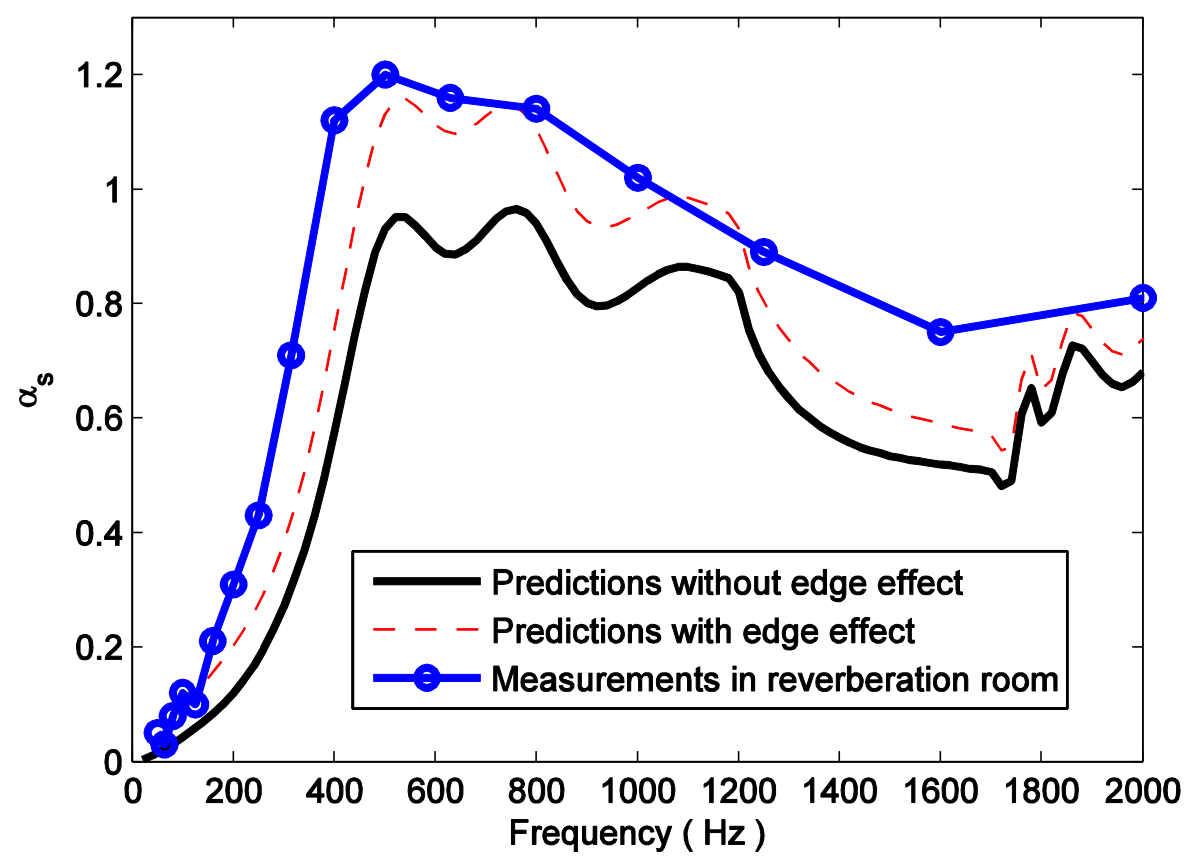

Figure 16: Comparison of the measured and predicted sound absorption coefficients in diffuse field. 
The vibration effect of the MPP on the measured sound absorption coefficients is assessed numerically. The procedure for modeling the vibration effect in the MPP absorber array is described in [15]. Figure 17 shows the predicted normal sound absorption coefficients $(\theta=0)$ with the MPP being made of rigid plate, steel plate and aluminum plate respectively. In the latter two cases, the four edges of the MPP are assumed to be simply supported. It can be seen that the structural vibration of both steel and aluminum plates can cause extra peaks or dips on the absorption curves, but the overall effect of structural vibration is negligible especially when steel plate is used.

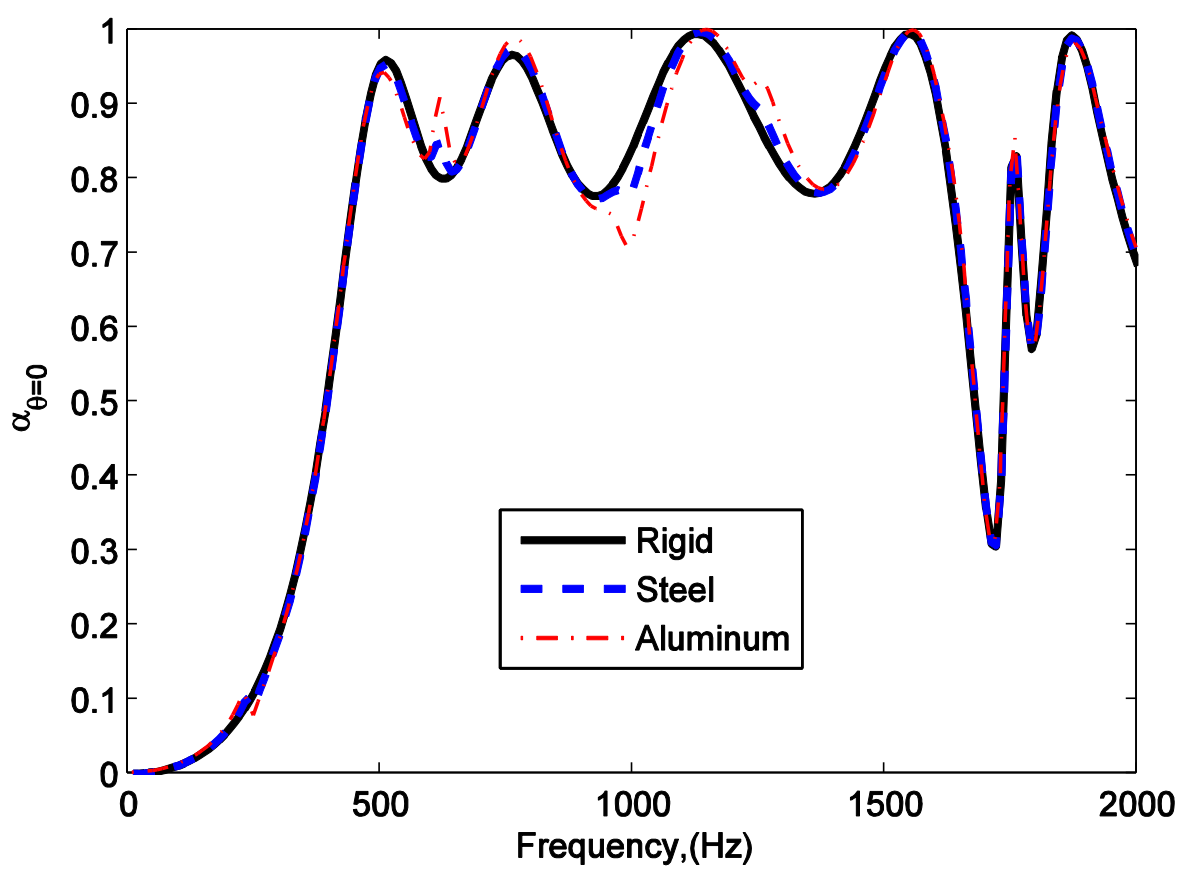

Figure 17: Predicted normal sound absorption coefficients of the MPP absorber array with the MPP being rigid plate, steel plate and aluminum plate respectively. Material properties: $E_{\mathrm{Al}}=7 \times 10^{10} \mathrm{~Pa}, E_{\mathrm{St}}=2 \times 10^{11} \mathrm{~Pa}, \rho_{\mathrm{Al}}=2700 \mathrm{~kg} / \mathrm{m}^{3}, \rho_{\mathrm{St}}=7850 \mathrm{~kg} / \mathrm{m}^{3}$.

\section{Conclusions}

The acoustic properties of a micro-perforated panel (MPP) absorber array are considered for oblique incidence and diffuse field. A 3D finite element model is developed to simulate the acoustic behaviors of the MPP absorber array. The diffuse field sound absorption coefficients of a prototype MPP absorber array are measured in 
a reverberation room and compared with the predictions. The following conclusions are made:

(1) The acoustic response of one resonator (i.e., one sub-cavity and the covering MPP) is influenced by all other resonators in the MPP absorber array. So, the sound absorption coefficient of the MPP absorber array can be noticeably different from that of one basic module tested in an impedance tube. For normal incidence, it is observed that the parallel absorption mechanism works when the cavity width is less than the acoustic wavelength of interest, which compares favorably with the result in one basic module in which the parallel absorption mechanism fails to work if the cavity width is larger than half wavelength.

(2) At oblique incidence, the equivalent acoustic impedance of the MPP decreases as the incidence angle increases, which changes the acoustic impedance matching conditions in the MPP absorber array. Consequently, the sound absorption performance of the MPP absorber array changes as a function of the incidence angle, and severe performance drop occurs at nearly grazing incidence.

(3) At oblique incidence, the parallel absorption mechanism breaks down at lower frequency than at normal incidence due to the non-compactness of the resonating MPP absorber. It is observed that the parallel absorption mechanism breaks down when the time delay of the incident wave across the resonating MPP absorber is comparable to or larger than $\pi / 2$. The effect of the azimuthal angle on the sound absorption is found to be insignificant.

(4) The measured sound absorption coefficients in a reverberation room compare well with the simulated results except that the experimental results are higher than the predictions on the whole. One major source of the extra sound absorption in the reverberation experiment is the edge effect of the finite test specimen, which is the most efficient around $500 \mathrm{~Hz}$. 


\section{Acknowledgments}

The work is supported by National Basic Research Program of China (973 Program: 2012CB720202). The first author thanks the Faculty of Engineering (HKU) for the postdoctoral fellowship.

\section{References}

[1] H.V. Fuchs, X. Zha, Micro-perforated structures as sound absorbers- A review and outlook. Acta Acustica united with Acustica 92 (2006) 139-146.

[2] H.V. Fuchs, X. Zha, Acrylic-glass sound absorbers in the plenum of the Deutscher Bundestag. Applied Acoustics 51 (1997) 211-217.

[3] G. Li, C.K. Mechefske, A comprehensive experimental study of micro-perforated panel acoustic absorbers in MRI scanners. Magnetic Resonance Materials in Physics, Biology and Medicine 23 (2010) 177-185.

[4] S.H. Park, A design method of micro-perforated panel absorber at high sound pressure environment in launcher fairings. Journal of Sound and Vibration 332 (2013) $521-535$.

[5] M.Q. Wu, Micro-perforated panels for duct silencing. Noise Control Engineering Journal 45 (1997) 69-77.

[6] S. Allam, M. Abom, A new type of muffler based on microperforated tubes. Journal of Vibration and Acoustics 133 (2011) 1-8. DOI: 10.1115/1.4002956

[7] D.Y. Maa, Micorperforated-Panel wideband absorbers. Noise Control Engineering Journal 29 (1987) 77-84.

[8] D.H. Lee, Y.P. Kwon, Estimation of the absorption performance of multiple layer perforated panel systems by transfer matrix method. Journal of Sound and Vibration 278 (2004) 847-860. 
[9] T. Bravo, C. Maury, C. Pinhede, Enhancing sound absorption and transmission through flexible multi-layer micro-perforated structures. Journal of the Acoustical Society of America 134 (2013) 3663-3673.

[10] Y.Y. Lee, EWM Lee, C.F. Ng, Sound absorption of a finite flexible micro-perforated panel backed by an air cavity. Journal of Sound and Vibration 287 (2005) 227-243.

[11] D. Chang, B. Liu, X. Li, An electromechanical low frequency panel sound absorber. Journal of the Acoustical Society of America 128 ( 2010) 639-645.

[12] X. Zha, J. Kang, T. Zhang, X. Zhou, H.V. Fuchs, Application approach for microperforated panel sound absorbers. Acta Acustica 19 (1994) 258-265.

[13] P. Wang, M.Q. Wang, Y.S. Liu, J.F. Zhang, H.X. Luan, Study on the Parallel Microperforated Panels. Piezoelectrics \& Acoustooptics 30 (2008) 489-491.

[14] M. Yairi, K. Sakagami, K. Takebayashi, M. Morimoto, Excess sound absorption at normal incidence by two microperforated panel absorbers with different impedance. Acoustical Science and Technology 32 (2011) 194-200.

[15] C.Q. Wang, L.X. Huang, On the acoustic properties of parallel arrangement of multiple micro-perforated panel absorbers with different cavity depths. Journal of the Acoustical Society of America 130 (2011) 208-218.

[16] M. Toyoda, R.L. Mu, D. Takahashi, Relationship between Helmholtz-resonance absorption and panel-type absorption in finite flexible microperforated-panel absorbers. Applied Acoustics 71 (2010) 315-320.

[17] D.Y. Maa, Potential of microperforated panel absorber. Journal of the Acoustical Society of America 104 (1998) 2861-2866.

[18] J.B. Keller, D. Givoli, Exact non-reflecting boundary conditions. Journal of Computational Physics 82 (1989) 172-192. 
[19] T. T. Wolde, Measurements on the edge-effect in reverberation rooms. Acustica 18 (1967) 207-212.

[20] D. E. Goldberg, Genetic Algorithms in Search, Optimization and Machine Learning, Addison-Wesley Longman, Boston, 1989.

[21] International Organization for Standardization ISO 354, Acoustics-measurement of sound absorption in a reverberation room, 2003.

[22] P.M. Morse, R.H. Bolt, Sound waves in rooms. Reviews of Modern Physics 16 (1944) 69-150.

[23] P.E. Sabine, What is measured in sound absorption measurements. Journal of the Acoustical Society of America 6 (1935) 239-245.

[24] Y. Kawai, H. Meotoiwa, Estimation of the area effect of sound absorbent surfaces by using a boundary integral equation. Acoustical Science and Technology 26 (2005) 123-127. 


\section{List of Figure Captions}

Figure 1: Configuration of the MPP absorber array. (a) Schematic of the partitioning of the backing cavity in one basic module; (b) Theoretical model of the MPP absorber array. The dashed lines represent the additional virtual duct for modeling the exterior sound field of the MPP absorber array.

Figure 2: Comparison of the predicted normal incidence $(\theta=0)$ absorption coefficients between configurations with "periodic" and "rigid" boundary conditions on the four sides of the virtual duct.

Figure 3: Illustration of the equivalent dimensions of the basic module arranged in a periodically repeating pattern. (a) Two adjacent basic modules in a large panel. Each module consists of four sub-cavities with different depths. The dashed lines represent the equivalent basic modules with reduced size. (b) Four types of equivalent basic modules owing to the geometry symmetry.

Figure 4: Variation of the normal incidence sound absorption coefficients of the MPP absorber array with respect to the geometrical dimension of backing cavity.

Figure 5: Variation of sound absorption coefficients at different incidence angles with $\beta=90^{\circ}$ and $L_{x}=L_{y}=100 \mathrm{~mm}$.

Figure 6: Comparison of sound absorption coefficients at different incidence angles with $\beta=90^{\circ}$ and $L_{x}=L_{y}=100 \mathrm{~mm}$.

Figure 7: The effective acoustic resistance of the MPP at different incidence angles. The effective acoustic resistance is estimated as four times the acoustic resistance calculated by Eq. (4). 
Figure 8. Variation of the oblique incidence sound absorption coefficients with respect to the geometrical dimensions of the backing cavity. $\theta=45^{\circ}$ and $\beta=90^{\circ}$.

Figure 9: Variation of sound absorption coefficients at different azimuthal angles with $\theta=45^{\circ}$. (a) $L_{x}=L_{y}=100 \mathrm{~mm}$; (b) $L_{x}=L_{y}=200 \mathrm{~mm}$.

Figure 10: Predicted diffuse field sound absorption coefficients for different geometrical dimensions of the backing cavity. The oblique incidence absorption coefficient for $L_{x}=L_{y}=150 \mathrm{~mm}$ at $\theta=45^{\circ}$ is shown for reference.

Figure 11: Oblique incidence sound absorption of parallel arrangement of two MPP absorbers with different cavity depths (100 mm and $25 \mathrm{~mm}$ ). (a) Schematic of the 2D configuration; (b) Sound absorption coefficients at different incidence angles.

Figure 12: Distributions of the sound intensity vectors below and above the breakdown frequency $(f=1050 \mathrm{~Hz})$ of the parallel absorption mechanism. The arrow length indicates the relative magnitude of sound intensity.

Figure 13: Sound absorption coefficients of a conventional uniform MPP absorber predicted by three models. Incidence angle $\theta=45^{\circ}$, cavity depth $D=100 \mathrm{~mm}$. Other parameters are given in Table 1.

Figure 14: Predicted oblique incidence sound absorption coefficients of the four-cavity MPP absorber array specified in Table 1. $\theta=45^{\circ}$ and $\beta=90^{\circ}$.

Figure 15: Photo of the large test specimen in the reverberation chamber. The large test specimen consists of 72 pieces of square samples.

Figure 16: Comparison of the measured and predicted sound absorption coefficients in diffuse field. 
Figure 17: Predicted normal sound absorption coefficients of the MPP absorber array with the MPP being rigid plate, steel plate and aluminum plate respectively. Material properties: $E_{\mathrm{Al}}=7 \times 10^{10} \mathrm{~Pa}, E_{\mathrm{St}}=2 \times 10^{11} \mathrm{~Pa}, \rho_{\mathrm{Al}}=2700 \mathrm{~kg} / \mathrm{m}^{3}, \quad \rho_{\mathrm{St}}=7850 \mathrm{~kg} / \mathrm{m}^{3}$. 